\title{
A Heliospheric Imager for Deep Space: Lessons Learned from Helios, SMEI, and STEREO
}

\author{
B.V. Jackson • A. Buffington • P.P. Hick • M.M. Bisi • \\ J.M. Clover
}

Received: 11 December 2009 / Accepted: 15 May 2010 / Published online: 12 June 2010

(C) The Author(s) 2010. This article is published with open access at Springerlink.com

\begin{abstract}
The zodiacal-light photometers on the twin Helios spacecraft, the Solar Mass Ejection Imager (SMEI) on the Coriolis spacecraft, and the Heliospheric Imagers (HIs) on the Solar-TErrestrial RElations Observatory (STEREO) twin spacecraft all point the way to optimizing future remote-sensing Thomson-scattering observations from deep space. Such data could be provided by wide-angle viewing instruments on Solar Orbiter, Solar Probe, or other deep-space probes. Here, we present instrument specifications required for a successful heliospheric imager, and the measurements and data-processing steps that make the best use of this remote-sensing system. When this type of instrument is properly designed and calibrated, its data are capable of determining zodiacal-dust properties, and of threedimensional reconstructions of heliospheric electron density over large volumes of the inner heliosphere. Such systems can measure fundamental properties of the inner heliospheric plasma, provide context for the in-situ monitors on board spacecraft, and enable physicsbased analyses of this important segment of the Sun-spacecraft connection.
\end{abstract}

Remote Sensing of the Inner Heliosphere Guest Editors: M.M. Bisi, and A.R. Breen.

B.V. Jackson $(\bowtie) \cdot$ A. Buffington · P.P. Hick · M.M. Bisi · J.M. Clover

Center for Astrophysics and Space Sciences, University of California, San Diego, 9500 Gilman Drive \#0424, La Jolla, 92093-0424 CA, USA

e-mail: bvjackson@ucsd.edu

A. Buffington

e-mail: abuffington@ucsd.edu

J.M. Clover

e-mail: jclover@ucsd.edu

M.M. Bisi

Institute of Mathematical and Physical Sciences, Aberystwyth, Ceredigion SY23 3BZ, Wales, UK e-mail: Mario.Bisi@aber.ac.uk

P.P. Hick

San Diego Supercomputer Center, University of California, San Diego, 9500 Gilman Drive \#0505, La Jolla, 92093-0505 CA, USA

e-mail: pphick@ucsd.edu 
Keywords Instrumentation and data management · Coronal mass ejections, interplanetary $\cdot$ Solar wind disturbances $\cdot$ Plasma physics

\section{Introduction}

Heliospheric remote-sensing observations provide one of very few means of observing structures in the solar wind between the time they leave the immediate solar environment and the time when they arrive and are measured by in-situ spacecraft instruments near Earth or in deep space. These remote-sensing data probe the global extent of the solar wind over a large range of solar elongations. They also extend across the high-latitude regions (the solar poles), which are difficult to access by other means. Past research has extensively used heliospheric remote sensing to study the physics of structures in the solar wind as they move out into the heliosphere.

Before spaceborne instruments made heliospheric imaging from remote-sensing data possible, interplanetary scintillation (IPS) observations of meter-wavelength intensity variations from point radio sources were used for heliospheric remote-sensing studies. IPS has long been used to measure small-scale $(\sim 200 \mathrm{~km})$ density variations along the line of sight to a radio source (see, e.g., Hewish, Scott, and Wills, 1964; Ananthakrishnan, Coles, and Kaufman, 1980). IPS observations taken using the Cambridge IPS array in the UK (Houminer, 1971), show structures that can be classified as either corotating or detached from the Sun (Gapper et al., 1982; Hewish and Bravo, 1986; Behannon, Burlaga, and Hewish, 1991). More recently, IPS data used in conjunction with tomographic threedimensional (3D) reconstruction techniques have shown the global shapes of heliospheric density and velocity structures including corotating regions and coronal mass ejections (CMEs) or their interplanetary counterparts (ICMEs) in the solar wind (see, e.g., Jackson et al., 1998; Kojima et al., 1998; Asai et al., 1998; Jackson, Hick, and Buffington, 2002; Jackson et al., 2003; Jackson and Hick, 2005; Tokumaru et al., 2007; Tokumaru, Kojima, and Fujiki, 2010; Bisi et al., 2007, 2008a, 2008b, 2009a, 2009b; Jackson et al., 2008a, 2008b, 2009, 2010). These tomographic analyses match in-situ spacecraft measurements from near-Earth spacecraft fairly well. Since they measure solar wind structures prior to their reaching Earth, they have been used to forecast solar wind conditions at Earth in near real time for over a decade at the Solar Terrestrial Environment Laboratory (STELab), Nagoya, Japan and the University of California, San Diego (UCSD), websites http://stesun5.stelab.Nagoya-u.ac.jp/index-e.html, and http://ips.ucsd.edu, respectively.

In the mid 1970s, spaceborne instruments first began to view the solar corona (Tousey, 1973); and shortly thereafter "discovered" CMEs (MacQueen et al., 1974; Koomen et $a l ., 1975)$ as the manifestations of the coronal depletions and changing mass measured before in ground-based coronagraph observations (Hansen et al., 1971, 1974). The first truly heliospheric white-light Thomson-scattering observations of CMEs (Richter, Leinert, and Planck, 1982) were obtained with the zodiacal-light photometers (Leinert et al., 1975) on the Helios spacecraft (Porsche, 1984). The analyses of these data using a variety of imaging, perspective viewing, and tomographic techniques demonstrating that Thomson-scattering observations were possible to large elongations (Jackson, 1985a, 1985b; Jackson and Leinert, 1985; Jackson et al., 1985) advanced the NASA proposal for the Large Angle and Spectrometric COronagraph (LASCO) C3 instrument that viewed to a then-unprecedented $7.5^{\circ}$ elongation (Brueckner et al., 1995). The analyses from Helios and following observations from C3 spurred development of the Solar Mass Ejection Imager (SMEI) instrument (Eyles et al., 2003; Jackson et al., 2004), and the Solar-TErrestrial RElations Observatory 
(STEREO) spacecraft Heliospheric Imager (HI) instruments (see, e.g., Eyles et al., 2009). For lessons learned in this article, we use the analyses from the Helios, SMEI, and STEREO white-light heliospheric imaging instrumentation.

Section 2 describes the Helios, SMEI, and STEREO HI data sets. Section 3 explains the motivation for obtaining precisely-calibrated heliospheric Thomson-scattering observations, and describes the difficulties that need to be overcome to extract these from the Helios photometer data and, by extension, from the SMEI white-light data. Section 4 discusses how a better instrument could be built and used for these same analyses, and finally Section 5 presents our conclusions.

\section{The Helios, SMEI and HI Imaging Data Sets}

Understanding the difficulties in measuring Thomson-scattered light down to the level needed to map heliospheric structures near Earth requires an assessment of the signal levels involved. Thomson-scattered signals must be separated from many other sources of diffuse light: background light from the Sun; zodiacal light; and starlight, either individually as bright point sources or collectively as a mottled contribution to the diffuse sky brightness (Figure 1). Here, one S10 is the sky brightness of one 10th magnitude solar-type star spread over a square degree (Cox, 2000). Heliospheric structures are viewed by scattered sunlight, which retains its same spectral characteristics. Since these structures typically cover many degrees of sky, and are detected and calibrated relative to background stars, the S10 unit is an ideal comparative measure for subsequent analysis of Thomson-scattering brightness. In near-Earth orbit, reflected sunlight from the Moon or Earth can scatter into the field of view; contributions from atmospheric ram glow, aurorae, and light from the geocorona can obscure the Thomson-scattering brightness (for a summary of these signals, see Jackson et al., 2004, and references therein).

When evaluating heliospheric and instrumental stray-light contributions in terms of S10 surface brightness relative to the total Sun in S10s (Figure 1, right-hand scale), the Sun's total brightness (in a fashion similar to stars) is spread out over one square degree, and near Earth

Figure 1 Surface brightness versus solar elongation for zodiacal and star light (from Allen's Astrophysical Quantities, Cox, 2000) and of expected CME brightness from observations using the Helios photometers and SMEI (also see footnote 1). Also shown is a calculation of an ambient heliospheric medium having a density of $10 \mathrm{~cm}^{-3}$ at $1 \mathrm{AU}$ and an inverse-square density drop-off with solar distance that matches the bright CME response. The SMEI stray-light value is from Buffington, Jackson, and Hick (2005), the STEREO HI-2 upper limit is from Eyles et al. (2009).

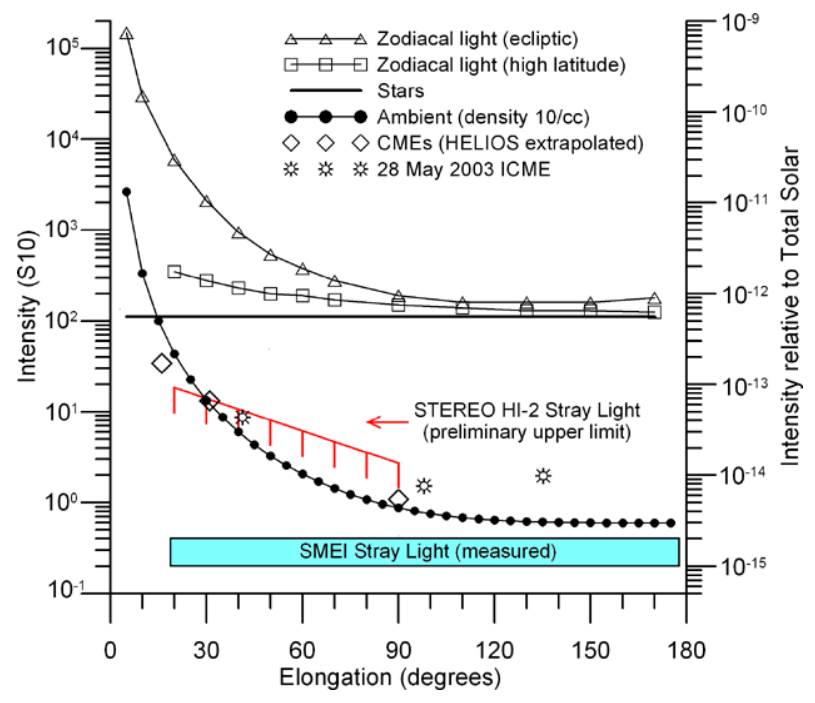


this decreases the Sun's surface brightness by a factor of $0.223 .{ }^{1}$ When the observer is closer to the Sun, this fraction becomes larger, as does the brightness of the heliospheric structures (see Section 4). The Sun is the equivalent of $\sim 2 \times 10^{14} \mathrm{~S} 10$ units when viewed from Earth by SMEI. A fundamental limit to diffuse-light photometry is set by photon counting statistics; a good instrument design reduces other noise sources down to or below this level. This limit depends upon the optics and scanning configuration, spectral bandpass, and detector efficiency. The total detected photon count $N$ for a square degree of sky projected onto the detector surface (see, e.g., Jackson et al., 1989) in Earth orbit is

$$
\log _{10} N=6.15-0.4 m+\log _{10} A \cos \theta+\log _{10} \Delta t
$$

where $A$ is the aperture area in $\mathrm{cm}^{2}, \theta$ the incident-light angle relative to the aperture normal, $\Delta t$ the integration time in seconds, and $m$ the brightness "magnitude" in a square degree evaluated for the instrument's particular bandpass. Here, the constant 6.15 includes a bandpass efficiency for the combination of detector and optics of roughly $20 \%$. Furthermore, a detector design requires that the total sky brightness signal $m$ must be determined well enough throughout the field of view. This will allow the background contribution to be removed sufficiently well (see Section 3) to be able to isolate the faint Thomson-scattered signal with sufficient precision for subsequent analysis.

The first truly remote-sensing heliospheric-imaging white-light data came from the zodiacal-light photometer experiments on the twin Helios spacecraft (Leinert et al., 1975) (Figure 2). There were two Helios spacecraft, Helios 1 was launched in December 1974, Helios 2 in January 1976. The Helios photometers on board these spacecraft were designed to map the brightness of the zodiacal-dust cloud to an unprecedented precision from their unique orbits (Leinert et al., 1975, 1981), which carried the spacecraft from 0.3 to $1.0 \mathrm{AU}$ in the ecliptic plane over a six-month time interval. With a downlink data rate of only one bit per second, these photometer systems mapped the sky in 65 heliographic locations at $16^{\circ}$, $31^{\circ}$, and $90^{\circ}$ ecliptic latitude, in three color bands, using a rotating filter wheel with three polarization orientations and a clear filter. The total sample interval extended over approximately five hours. Because absolute photometry was a goal for these Helios instruments, the photometers and the Helios spacecraft were designed to keep stray light to a minimum, where other instruments up to that time had failed (see Leinert and Klüppelberg, 1974). The Helios photometers had no discernable stray-light contribution, although attempts were made to find an amount by slightly tilting the spacecraft from its spin axis perpendicular to the ecliptic plane. This allowed sunlight to enter into the blackened skirt of the spacecraft (see Figure 2), and presumably into the ends of the Helios photometer tubes at certain times during the one-second spacecraft revolution period (Leinert et al., 1981).

The Helios photometers measured the zodiacal-light brightness in the inner heliosphere in three colors and polarized light with unprecedented precision (see, e.g., Leinert, Richter, and Planck, 1982; Leinert and Pitz, 1989). The Helios photometer systems detected the brightness of "plasma clouds" (Leinert, Richter, and Planck, 1982) that were traced in several examples by Richter, Leinert, and Planck (1982) to CMEs observed by the Solwind coronagraph (Sheeley et al., 1980). The Helios spacecraft spin axis was usually kept perpendicular to the ecliptic plane with the Helios 1 photometers viewing to the south, and

\footnotetext{
${ }^{1}$ We note that the right-hand scale of Figure 1 is determined in the context of SMEI which views in the red rather than visual light. This differs from the $\mathrm{B} / \mathrm{B}_{\text {sun }}$ used in coronagraph observations (Socker et al., 2000; as defined by LASCO observations, Morrill et al., 2006; and see a discussion in Buffington et al., 2007). In this alternate method of brightness ratio measurement, a unit sky pixel on the image is compared with the brightness of the mean Sun were it to fall on this pixel assuming the Sun more than overlaps the unit pixel.
} 
Figure 2 The Helios spacecraft. The Helios spacecraft photometers are shown nestled behind the blackened skirt of the spacecraft.

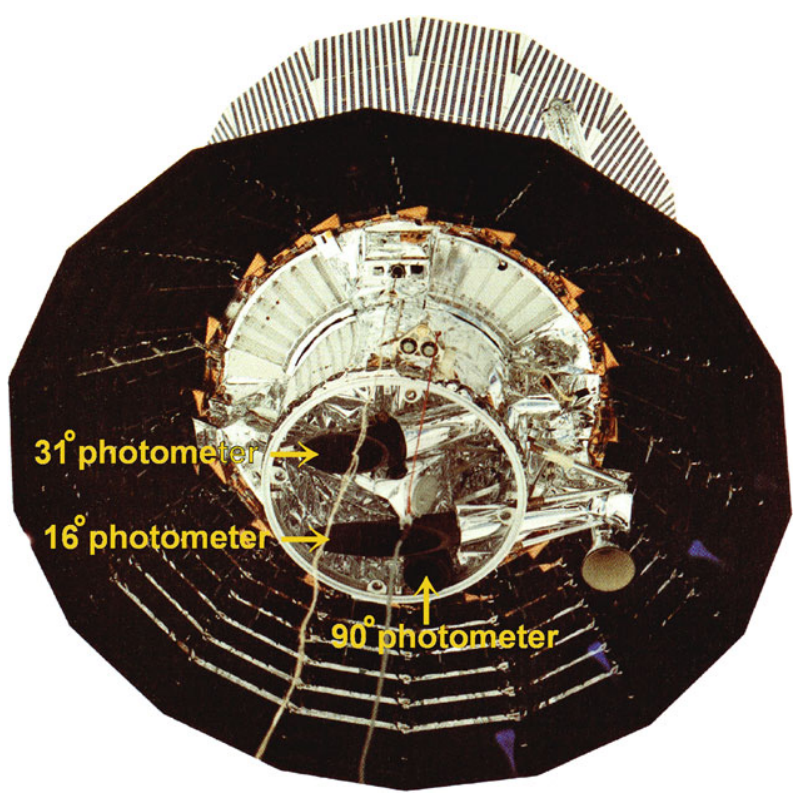

those of Helios 2 viewing north. Nevertheless, the Helios photometer coverage of the sky retained enough spatial information to provide $\mathrm{CME}$ images over nearly half a hemisphere (Jackson, 1985a, 1985b; Jackson and Leinert, 1985) from its non-Earth viewpoint. This information was used to provide images and to track a halo CME (as observed from Earth) outward along the Sun-Earth line from the perspective of the two Helios spacecraft until it produced a geomagnetic storm (Jackson, 1985a), and to determine perspective information about the 3D shapes of CMEs (see, e.g., Jackson et al., 1985; Jackson, Rompolt, and Švestka, 1988). These same white-light observations were also used to view corotating heliospheric structures and to measure their outflow and persistence (Jackson, 1991). Although a superb instrument for measuring zodiacal light, the low data rates from the Helios photometers and their poor spatial coverage allowed only a rudimentary measurement of the wealth of fine structure present in the plasma heliosphere.

Building on this instrumentation and these ideas, SMEI (Figure 3) (Eyles et al., 2003; Jackson et al., 2004) was designed to image nearly the entire sky in visual light during each 102-minute Earth orbit, and to map large-scale variations in heliospheric electron densities around Earth. The SMEI design required that the instrument provide a 3-sigma signal for Thomson-scattered light in a square degree of sky $90^{\circ}$ from the Sun for a single orbit exposure, and this specification was shown to be adequately met (Buffington et al., 2006). SMEI provides roughly an order of magnitude improved angular and photometric resolution compared with Helios, and a three-fold faster cadence for full-sky maps. Designed for deployment on the Wind spacecraft (Ogilvie and Desch, 1997), SMEI was initially conceived to operate on a rapidly-rotating satellite in deep space (Jackson et al., 1989). In this configuration, the SMEI detectors would have counted individual photons and integrated their totals over time much as the Helios photometers had. Various constraints and lack of funding curtailed this initial effort for Wind. After the Helios spacecraft were launched, cooled, low-readout-noise CCD detectors became available and were flown into space. With no deep-space satellite bus available to provide an instrument launch, and with a large data downlink available from near Earth, SMEI was redesigned with a CCD detector and baffles 


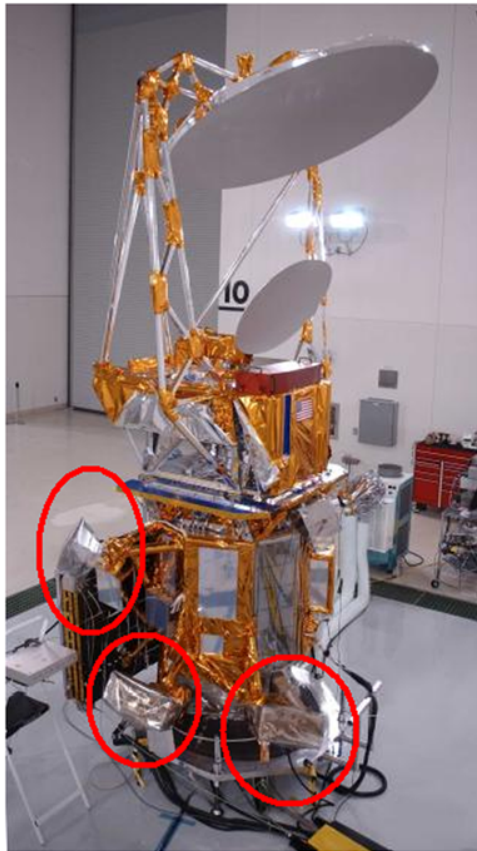

(a)

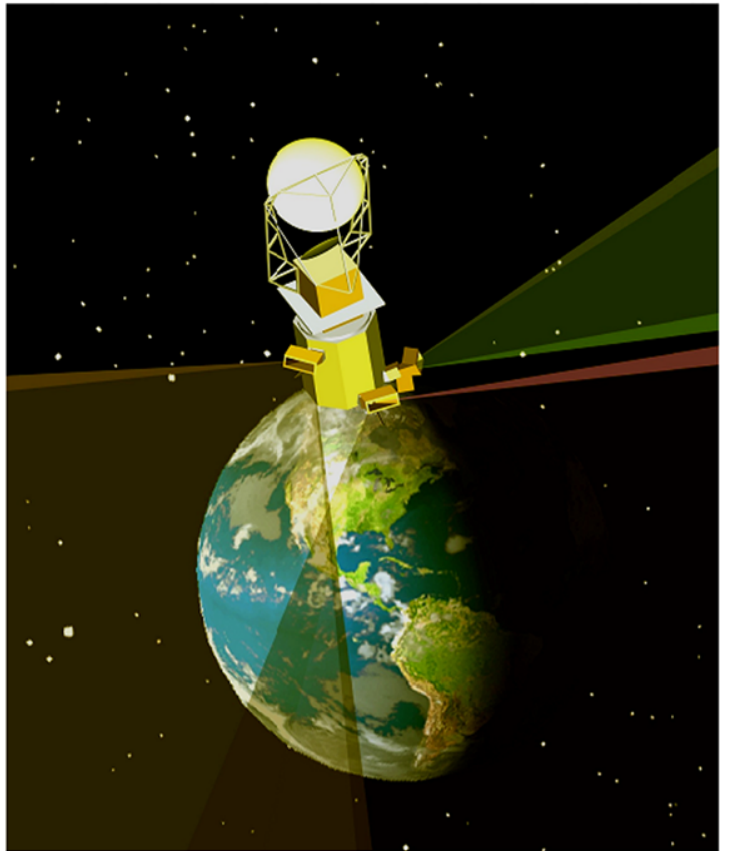

(b)

Figure 3 (a) The Coriolis spacecraft with the Solar Mass Ejection Imager (SMEI) instrument (Jackson et $a l ., 2004)$ and the Windsat antenna prior to launch from Vandenberg AFB. The three SMEI camera baffles (circled) are seen on the lower portion of the spacecraft. (b) SMEI in its polar orbit at $840 \mathrm{~km}$ with an orbital inclination of $98^{\circ}$. SMEI looks away from the Earth at $30^{\circ}$ from the local horizontal to avoid sunlight reflected from the Earth and from the Windsat antenna. The combined fields of view of the three cameras (shown as shaded cones) cover nearly all the sky.

for Earth orbit using the Air Force Space Test Program P91-1 spacecraft. When resources proved unavailable to include the instrument on P91-1, the SMEI design was placed on hold until the University of Birmingham, UK, with a contribution of NASA funding, joined the effort with contributions of manpower and money starting in 1995.

With this design change for SMEI to operate in Earth orbit, a new primary consideration was that this orbit be high enough to stay above the atmospheric glows previously reported from lower similar instruments that caused them to fail. Variable stray-light signals (with changing spacecraft orientation) from the Sun, Earth, Moon, and from the spacecraft bus itself needed to be kept below the Thomson-scattering signal level. For the Helios photometers, SMEI, and the HI instruments, stray light is controlled by an external baffle. For a practical design that could be flown on an existing spacecraft bus, the SMEI baffle needed to fit within approximately a $30 \mathrm{~cm}$ length rather than the $100 \mathrm{~cm}$ length available for Helios. The main advantage of an imaging system over the Helios photometers, however, is that imaging enables identification of point objects (mostly stars) in the instrument field of view. Helios required that each stellar signal above about the fourth magnitude within the photometer field and color band be known and identified beforehand. This was acceptable for the photometric precision required for the zodiacal-light brightness, and the relatively small fields of view (and small numbers of stars) viewed by each Helios photometer, but this becomes a far more difficult task if stars must be removed down to the Thomson-scattering signal level at $1 \mathrm{AU}$ over the whole sky (see Figure 1). 
SMEI, now into its seventh year of operation, is the first true heliospheric imaging system to track CMEs outward from the Sun until they arrive at Earth (Jackson et al., 2004; Tappin et al., 2004). The photometric signals from SMEI allow 3D reconstructions and modeled 2D images (see, e.g., Jackson et al., 2006, 2008a, 2009; Buffington et al., 2008, 2009a; Bisi et al., 2008a). The SMEI team has produced significant scientific results directly from the 2D sky map orbit differences (see, e.g., Tappin et al., 2004; Reiner et al., 2005; Tappin, 2006; and Webb et al., 2006, 2009b; Tappin and Howard, 2009). One of the most notable serendipitous discoveries using the SMEI data has been the measurement of high altitude aurora (Mizuno et al., 2005). Removal of zodiacal light to an unprecedented precision has led to the precise characterization of the Gegenschein brightness (Buffington et al., 2009a). The SMEI images have also been used to measure solar wind speeds from comet tails with an unprecedented precision (Buffington et al., 2008). There are now at least three near-real time data pipelines operating (including one at UCSD) to image SMEI data in order to forecast CME arrival at Earth. SMEI has proven to be a robust Earth-orbiting instrument and as a pathfinder mission has been extremely successful. Auroral signals over the north and south poles of Earth, and high energy particle noise during South Atlantic Anomaly and in auroral oval passes, produce significant amounts of unwanted noise in the SMEI orbit. Inadequate cooling of the sun-pointing camera has also hastened the degradation of the sunward SMEI $\mathrm{CCD}$, and the images from this instrument camera. For solar studies, however, the single most-significant difficulty with the SMEI instrument is its inability to view close to the Sun in order to measure the onset of the plasma structures that originate there.

The twin STEREO spacecraft (see, e.g., Kaiser et al., 2008) Sun-Earth Connection Coronal Heliospheric Investigation (SECCHI) instrument suite (Howard et al., 2008) (Figure 4) was designed primarily to track CMEs all the way from the Sun along the Sun - Earth line to Earth. With two spacecraft, coronal detail can be viewed in stereo using simultaneous images when the spacecraft are near Earth and associated directly with solar surface features. At locations more distant from Earth the SECCHI coronagraph instruments in combination with the HIs (Harrison et al., 2008; Eyles et al., 2009) provide a near-continuous view of CMEs and other heliospheric structures as they move from their origin near the Sun to Earth. With the consideration of a Sun to Earth view as a design objective, the requirement that the

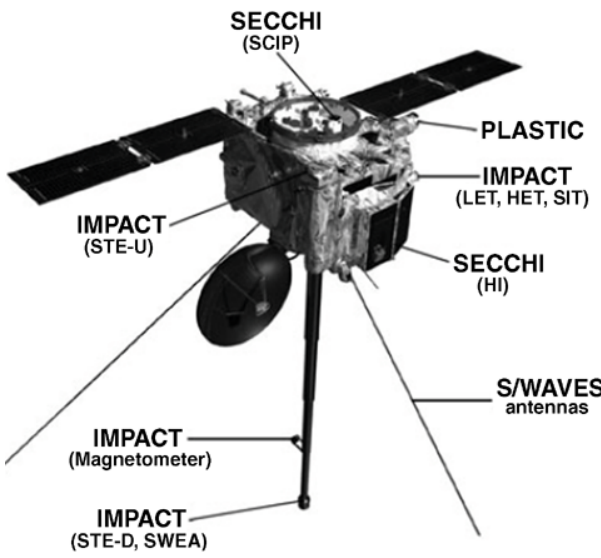

(a)

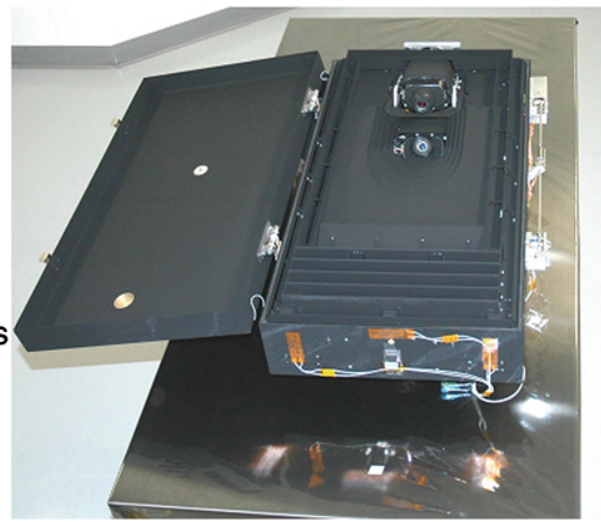

(b)

Figure 4 (a) One of the two STEREO spacecraft showing the instrument suite on board. Of particular interest, and similar in concept to SMEI, are the SECCHI HI-1 and HI-2 instruments (example shown in (b)). 
imager suite opening angle be at least the average CME width observed in coronagraphs, and from stray light and field-of-view considerations predicated by other instruments and spacecraft appendages on STEREO, the fields of view from the SECCHI HIs were limited in latitude and angular opening. The HI- 1 instruments have a field of view of $20^{\circ}$ beginning at $\sim 4^{\circ}$ from the solar limb, and the HI-2 instruments, a $70^{\circ}$ field of view beginning at $18.7^{\circ}$. This smaller field of view, with its orientation fixed relative to the Sun - Earth line, significantly reduces the difficulty of managing stray light variation in the $\mathrm{HI}$ images as compared with SMEI.

The SECCHI imaging suite has had high NASA visibility, and the HI instruments have performed extremely well since STEREO launch in 2006. Because there is significantly more coronal and heliospheric signal near the Sun, the HI-1 instruments can view fine detail in heliospheric structures that is then traced outward to $1 \mathrm{AU}$. The HI achievements include the detailed tracking of CMEs all the way from $4^{\circ}$ past $1 \mathrm{AU}$ in the ecliptic (see, e.g., Davis et al., 2009; Webb et al., 2009a), the measurement of fine structure present in corotating regions (see, e.g., Sheeley et al., 2008; Rouillard et al., 2008), and the measurement of the fine structure in a CME from the perspective views of both instruments until they reach Earth (Liu et al., 2010). The HI images have spawned a considerable number of modeling efforts aimed at understanding the 3D shapes of CMEs from the wealth of SECCHI instrument data (see, e.g., Vourlidas and Howard, 2006; Lugaz et al., 2008; Davis et al., 2009; Howard and Tappin, 2009). A good summary of HI achievements in the first two years of STEREO operation can be found in Harrison et al. (2009). The relatively small fields of view of the $\mathrm{HI}$ instruments, while very good at measuring remote structures in the ecliptic, do not work well to map a structure that impacts the viewing spacecraft, and are less able than Helios or SMEI to view the heliosphere globally. In addition, operated nominally, the HI instruments cannot directly measure the Archimedian spiral structure to the north and south, and map the interactions between structures that co-rotate and faster (or slower-moving) solar wind features.

\section{Motivation to Provide Precise Heliospheric Brightness Data}

To improve upon the observations available from the IPS and the Helios photometers, SMEI was conceived from the onset as an instrument capable of providing long-term measurements of heliospheric brightness with high precision, in order to determine the extent of, and to forecast, the arrival of heliospheric plasma structures near Earth. With precise measurements, the change of the heliospheric structure response along the line of sight (LOS) with elongation can be used to measure its distance from the observer. This is shown in a rudimentary way in Figure 5. The solar wind follows well-known physical principles in its outward flow. These physical principles have led to many different solar wind modeling efforts such as those using 3D kinematic models (i.e., Fry et al., 2003), and 3D magnetohydrodynamics (MHD) (see, e.g., Odstrcil and Pizzo, 1999; Odstrcil et al., 2004). Intensity IPS observations measure the amplitude variations of radio signals traveling through the heliosphere from small-scale inhomogeneities $(\sim 50-200 \mathrm{~km}$ in size $)$ in solar wind electron density that can be optically thick to the radio waves used to observe them. These smallscale electron density inhomogeneities provide only an uncertain proxy for solar wind bulk density (see, e.g., Jackson et al., 1998). On the other hand, heliospheric electron Thomsonscattering is a well-known process that is optically thin (Billings, 1966) and this, unlike intensity IPS, is a more direct measure of solar wind bulk density.

As heliospheric structures move outward from the Sun, spacecraft instrumentation shows to a first approximation that this flow is radial above about 15 solar radii. The material in 
Figure 5 Outward-flowing solar wind structure follows very specific physics as it moves away from the Sun.

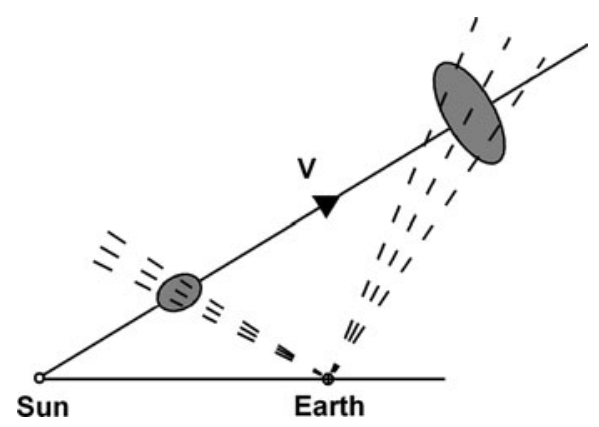

these structures changes shape to first order as in a "kinematic system" with mass and mass flux conserved as faster material catches up with slower, or slower recedes from faster. On average, however, the heliosphere expands at constant velocity, implying an $r^{-2}$ density fall-off with distance from the Sun. As the elongation of the outflowing material increases, it does not escape from view, but simply is viewed from a different perspective. For the IPS analyses the LOS weight function can be separated (approximately) from the amount of the density inhomogeneities present in the heliosphere. In the case of Thomson scattering, the LOS weighting can be separated from the density of the electrons at any given elongation (see, e.g., Jackson and Hick, 2002; Jackson et al., 2008b for a description of these weighting processes). These considerations led to using the change in brightness of heliospheric structures relative to the LOS to determine their position relative to the observer. Figure 5, coupled with Figure 6 provides a graphical overview of this process. The reader is referred to other articles (Jackson, 1985b; Jackson and Hick, 2002; Vourlidas and Howard, 2006; Howard and Tappin, 2009; Jackson et al., 2008b) for mathematical descriptions of the location of heliospheric material relative to the point of closest approach of the LOS to the Sun (or observer).

Other instrument techniques to determine the LOS extent and locations of coronal and heliospheric structures have been attempted. These utilize the difference between the LOS polarization brightness $(p B)$ and brightness weighting to give information about the LOS position of coronal structures relative to the point the LOS comes closest to the Sun (Munro, 1977; Moran and Davila, 2004, and references therein), and heliospheric structures (Jackson and Froehling, 1995). An instrument providing $p B$ measurements usually accomplishes this using a series of three rotating Polaroid filters, and thus has significantly greater weight and complexity than one that does not. For a heliospheric imager at $1 \mathrm{AU}$, photons collected over most of the sky number only in the hundreds per second per degree of sky, and $p B$ measurements decrease the amount of light by approximately an order of magnitude. Thus in general, unacceptably longer integration times would be required to achieve adequate photometric precision with this type of instrument. A practical instrument design that can provide this type of heliospheric imaging process has yet to be thoroughly explored. However, we note, that the ambiguity, whether a heliospheric structure is or is not beyond the point of closest approach of the LOS to the Sun, is removed at elongations $>90^{\circ}$ when using this technique (see Figure 6).

Measurements of the changing brightness of a heliospheric structure, as it moves across the sky, can be used to determine its distance from the observer. This requires that the background brightness can be determined accurately over the entire time period (or longer) that the structure is observed. This allows the background to be consistently removed, thus isolating the brightness due to the structure itself. Note that this cannot be achieved by "image 


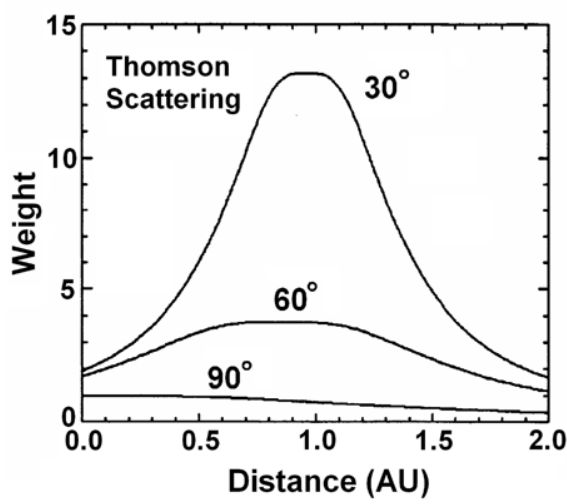

(a)

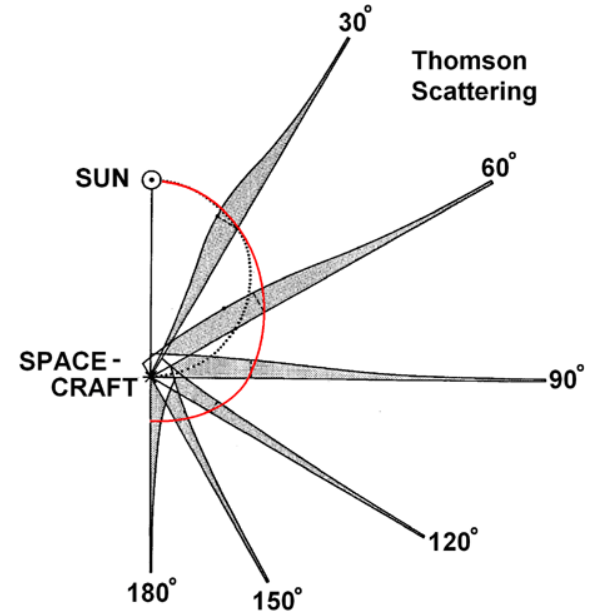

(b)

Figure 6 The weighting along the LOS at three elongations for electron Thomson scattering. (a) Distances to $2 \mathrm{AU}$ are included in these lines of sight outward from the observer. The "opening angle" of a view of the sky is included in the brightness measurement of a single electron per $\mathrm{cm}^{-3}$ along the LOS. The electron distance from the Sun decreases the line-of-sight brightness with solar elongation that is normalized to unity at $90^{\circ}$ elongation at the observer. (b) Relative-response distribution of the brightness contribution along the LOS for an $r^{-2}$ heliosphere. The point of closest approach to the line of sight to the Sun is marked by the dotted line. The red line shows the midpoint location of the LOS response (from Jackson, 1985b), and has been used in this and previous articles as a rough means to estimate the distance of structures beyond $90^{\circ}$ elongation.

differencing" where one image is simply subtracted from another. For CMEs viewed from Earth (where the motion of Earth is relatively slow compared to the solar wind speed), this implies that the CME must be measured together with the surrounding structures over the time span that it takes the ensemble of structures to flow from the Sun past Earth. For average solar wind speeds of $400 \mathrm{~km} \mathrm{~s}^{-1}$, this means the time it takes for material to flow from the solar surface out to about $2 \mathrm{AU}$, or over about eight days. For corotating structures a slightly longer time base (about 14 days) is needed to be able to discern these structures as they evolve moving across the sky from east to west of the Sun. For a tomographic inversion technique to be able to fully disentangle these features the Thomson-scattering signal needs to be determined with a precision of perhaps $10 \%$ or better. For less accurate data one very quickly loses the ability to deconvolve 3D heliospheric structures, and it is only possible to determine the maximum response of structures on successive image frames.

That all these criteria can be met has been amply shown by SMEI whose observations have been used to successfully reconstruct in-situ densities, observed near Earth, and at the STEREO spacecraft, both for the "quiet" background solar wind as well as for transient heliospheric structures (CMEs, corotating structures, and the density enhancements behind interplanetary Shocks; Bisi et al., 2008a; Jackson et al., 2008a, 2010, and references therein; see, e.g., Figure 7). The current 3D reconstructions of heliospheric structures available from the SMEI data use only about 1/50th of the information available on SMEI skymaps. Achieving considerably better spatial and temporal resolutions is currently limited by the available computer resources for SMEI data. 


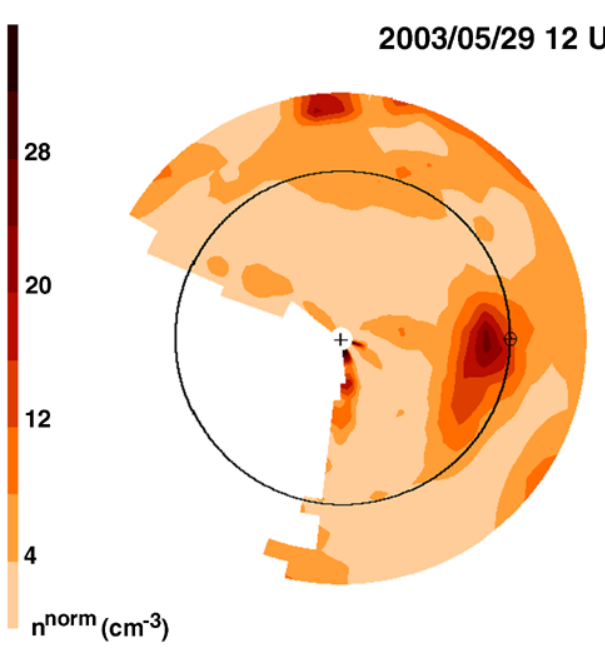

(a)

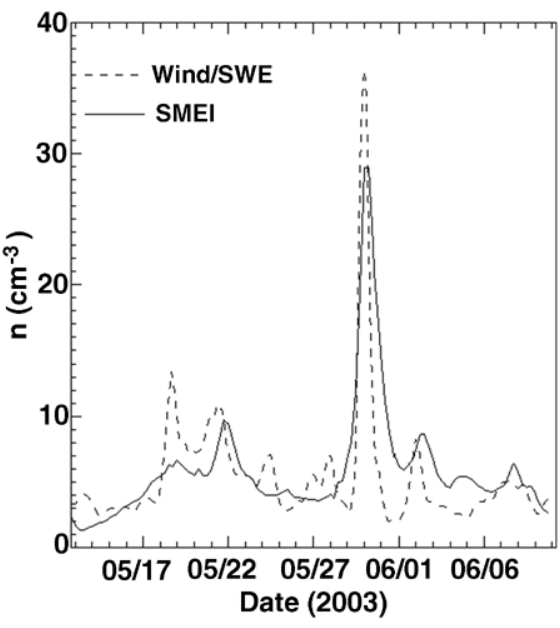

(b)

Figure 7 3D reconstruction of the 27-28 May 2003 halo ICME sequence of events. (a) An ecliptic-plane cut of the reconstructed density at the time when the CME reaches Earth, viewed from the North. The Sun is at the center marked with a + , an ellipse marks the Earth's orbital path, the Earth is shown by $\oplus$ (from Jackson et al. (2008b)). The density scale is given to the left. To best display the structure, an $r^{-2}$ density fall-off has been removed to scale densities to $1 \mathrm{AU}$. The main structure at Earth is associated with the halo CME sequence observed by LASCO on 27-28 May 2003. The density enhancement of the ICME hitting Earth in this event is more extensive to the east of the Earth than to the west. (b) Time-series plot of the density at Earth from the reconstruction and from Wind proton observations for the whole Carrington rotation that includes the ICME. The latter in-situ measurements are combined into 12-hour averages matching the temporal and spatial resolutions of the SMEI reconstruction. The correlation coefficient within six days of the ICME passage is 0.86 .

\section{Towards a Better Heliospheric Imager}

With the current analyses, it is clear that a system that holds variable stray light to a minimum, and consistently below the Thomson-scattering signal level is of primary importance. At 1 AU the signal level for bright CMEs approximates an ambient heliospheric medium having a density of $10 \mathrm{e}^{-} \mathrm{cm}^{-3}$ at $1 \mathrm{AU}$ and an inverse-square density drop-off with solar distance. At $90^{\circ}$ elongation, this implies a changing stray-light brightness per square degree on the detector that is below a few parts in $10^{-15}$ of the brightness of the Sun. It is desirable that this be kept constant, or at least modeled and removed down to this level, for the time it takes the transient heliospheric structure to pass through the instrument view (eight days to two weeks). Some background solar wind structures are fainter than the maximum sky brightness, and to measure these effectively as they move across the image, stray-light changes must be kept to an even lower level.

Sidereal-sky brightness adds noise to the system. A typical square degree of sky contains the equivalent brightness of 200 10th magnitude stars, and at least one 8th magnitude star. At $1 \mathrm{AU}$, this contribution is a factor of several hundred brighter than the Thomson-scattering signal at $90^{\circ}$. It does little good to finely resolve the sky and try to isolate these bright stellar signals since nearly every square degree of sky has one star that is nearly a factor of five brighter than the brightest heliospheric CMEs at these elongations. To enable removal of this contribution, the detector must therefore provide a reliable measurement of a star's brightness, no matter where it falls on the image plane. In addition steep gradients in brightness 
across the point spread function (PSF) or detector, or simply motion of the geometric-edge of the stellar image must not cause stars to have an apparent changing total brightness as these move across the detector. For this reason in SMEI, the PSF covers 50 pixels on the detector, with optics designed to create as bland a point image as the fast off-axis SMEI optics allow. The STEREO HI-2 instruments have a more circular PSF which covers $\sim 35$ detector pixels.

It is clearly desirable for heliospheric measurements that the detector view as much sky as possible. There are several reasons for this. The first is simply that with larger fields of view more heliospheric structures are observed. Given that a field of view that extends into the hemisphere away from the Sun provides more information close to the observer than does an instrument with a narrow field of view directed toward the Sun, observations in the antisolar hemisphere will help to extend in-situ measurements at the spacecraft away from it. An examination of Figure 6 shows why this is so. For lines of sight greater than $90^{\circ}$, the contribution from the LOS signal is at a maximum at the spacecraft. This becomes the most-steeply defined at $180^{\circ}$ opposite the Sun. With a smaller contribution from LOS segments distant from the observer, for a scientific instrument designed to extend structures near the observer to distances from it, this is a primary reason to provide observations into the hemisphere opposite the Sun. With SMEI, using signals from the hemisphere of sky beyond $90^{\circ}$ only, in-situ observations can be adequately reconstructed (see, e.g., Jackson et al., 2008a). Finally, the background sidereal brightness across the entire sky can be most accurately characterized using data from the anti-solar hemisphere because the Thomsonscattering and zodiacal-light signal is at a minimum there. The sidereal background sky thus determined can then be removed at other times from observations closer to the Sun where these signals are much stronger.

There is also a premium on viewing heliospheric structures very close to the Sun. For a single white-light instrument with a large field of view, this is difficult to accomplish because of the very large sky-brightness variation from near to far away from the solar surface. Add to this that with more light close to the Sun much finer angular resolutions become possible, it makes more sense to provide more than one instrument to view the regime close to and far away from the Sun (assuming that adding detectors, weight, and telemetry is not an issue). This was argued effectively for the STEREO SECCHI instruments. For an instrument viewing sky brightness from deep space at any distance from the Sun, the following Thomson-scattering principles hold.

i) The Thomson-scattering sky surface brightness at a given solar elongation in the sky falls off with Sun-observer distance $r$ as $r^{-3}$. Relative to the total solar brightness the fall-off is $r^{-1}$; thus as a spacecraft moves closer to the Sun, the Thomson-scattering sky gets somewhat brighter relative to the Sun.

ii) The $r^{-1}$ dependence on Sun-observer distance is valid for almost any heliospheric elongation from $0.1 \mathrm{AU}$ out to and beyond $1 \mathrm{AU}$.

The $r^{-1}$ dependence can be understood using Figure 8. In general, for most of the heliosphere above 10-20 solar radii, the solar wind neither accelerates nor decelerates. At constant velocity, this implies the solar wind density changes by $r^{-2}$ as it moves outward from the Sun. In Figure 8, observer B is twice the distance from the Sun as observer A. Both are viewing along a LOS with the same elongation angle from the Sun and the same opening angle. A sees a solar wind packet (indicated in blue). The same packet is shown when it has moved outward twice as far from the Sun (and now lies along the LOS from B). The electrons in this packet contribute a certain amount of light to the Thomson-scattering brightness when viewed from A or B. Each electron seen by B intercepts less light from the Sun by 
Figure 8 Graphical depiction of the brightness of the sky at two locations from the Sun.

Location $A$ is $1 / 2$ the distance of the Sun from B. The lines of sight at A and B have the same elongations and opening angle.

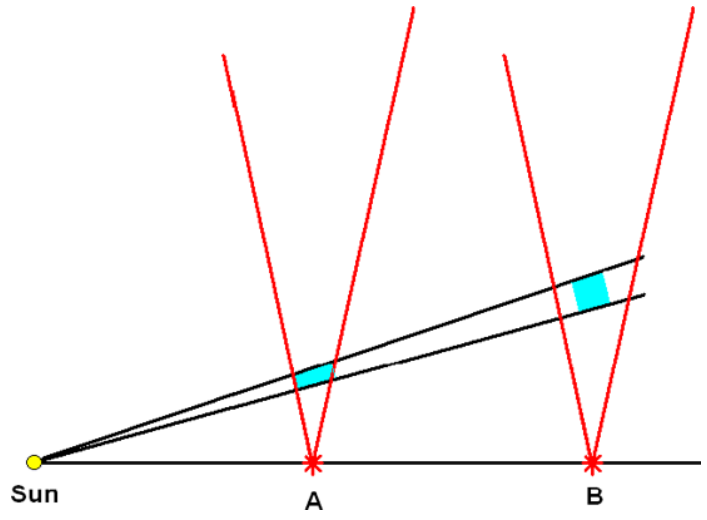

a factor of $r^{-2}$ (a factor of 1/4) as an electron seen by A. Because the scattering geometry (the angle Sun-electron-observer) is the same for A and B, electrons will re-radiate the same fraction of the incident light toward the observer. Since each electron at B is twice as far from the observer than at $\mathrm{A}$ its contribution to the observed brightness decreases by another factor of $1 / 4$. Thus, for each electron, the total contribution at $B$ is $1 / 16$ th the amount at $A$. However, since the opening angle is the same for A and B, two packets of outward-moving electrons now fit within the LOS at B. So the contribution from all electrons at B is $1 / 8$ th the amount at $\mathrm{A}$, resulting in a $r^{-3}$ dependence for the total Thomson-scattering brightness. At B the Sun is twice the distance of A; hence its apparent brightness is decreased by $1 / 4$ th from A. Thus the Thomson-scattering brightness at B normalized to the Sun's brightness is only $1 / 2$ of $\mathrm{A}$, resulting in a $r^{-1}$ dependence.

In Figure 9 we determine the brightness of the sky using the equations in Billings (1966), and knowing the numbers of heliospheric electrons from modeled data, the sky brightness is calculated by summing along the integral path out to a distance of 3 AU. Figure 9a plots the ratio of the integral brightness from the number of heliospheric electrons assuming a $r^{-2}$ solar electron density fall-off with $10 \mathrm{e}^{-} \mathrm{cm}^{-3}$ at $1 \mathrm{AU}$. We obtained the total solar brightness from the Cox (2000) value in visual-light. This value is not a surface brightness, and the limb darkening of a few percent at the spacecraft is not included (although limb darkening is included for the electrons from the Billings formulae). Figure $9 \mathrm{~b}$ plots this same ratio using the Cox values of heliospheric density. The Cox densities have about $10 \mathrm{e}^{-} \mathrm{cm}^{-3}$ at $1 \mathrm{AU}$, and the two heliospheric density distributions give approximately the same answer. The Cox densities show a slight increase in brightness in the lower left hand corner of Figure $9 \mathrm{~b}$ that shows where the $r^{-2}$ approximation does not hold because on average the solar wind is accelerating close to the Sun at that location in Figure $9 \mathrm{~b}$.

The most severe restriction placed on a heliospheric imager is the very large difference between the signal intensity and the brightness of the Sun, the latter of which will also illuminate the spacecraft bus and portions of the imager (Figures 1 and 9). This large range of brightness challenges, and usually precludes, direct laboratory testing except under very limited circumstances (see, e.g., Figure 10 adapted from Buffington, 2000). In a heliosphericimager design there are two important considerations in the elimination of stray light, the field of view and the "field of regard". The first is simply the region of the sky that falls on the image plane of the detector, while the second includes also the region outside this, which, because of its proximity to the field of view, must also not have extremely-bright objects present. For the Helios $90^{\circ}$ photometer, a SMEI camera unit, and the STEREO $\mathrm{HI}-2$, the former quantity is respectively, $3^{\circ}, 3^{\circ} \times 60^{\circ}$, and $70^{\circ}$. All of these designs em- 
Figure 9 Ratio of the visual sky brightness relative to solar brightness. Contours are $\log _{10}$ of respectively, (a) the $R^{-2}$ density model with $10 \mathrm{e}^{-} \mathrm{cm}^{-3}$ at $1 \mathrm{AU}$, and (b) the Cox (2000) density model.
Figure 10 Measured stray-light rejection from curved (radii $R=1.0 \mathrm{~m}, 0.5 \mathrm{~m}$ ) surface. Note the insensitivity of the performance to surface smoothness (from Buffington, 2000).

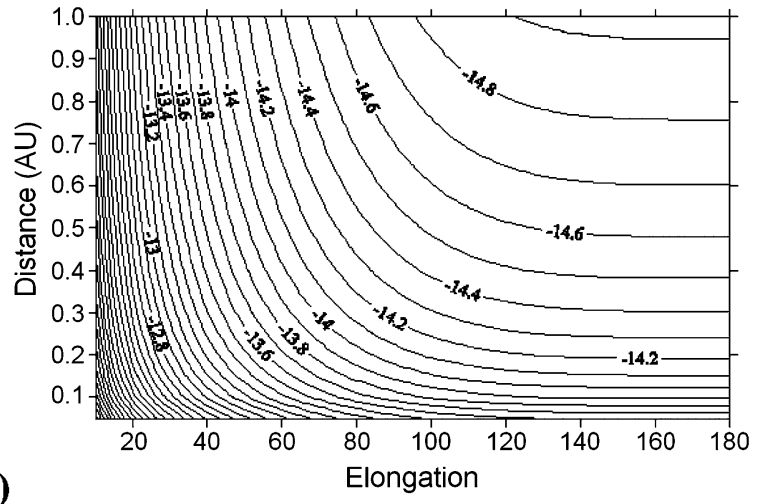

(a)

(b)

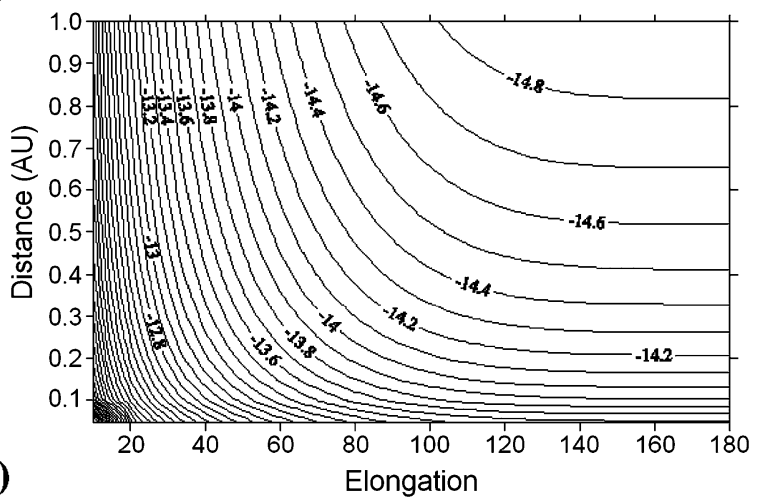

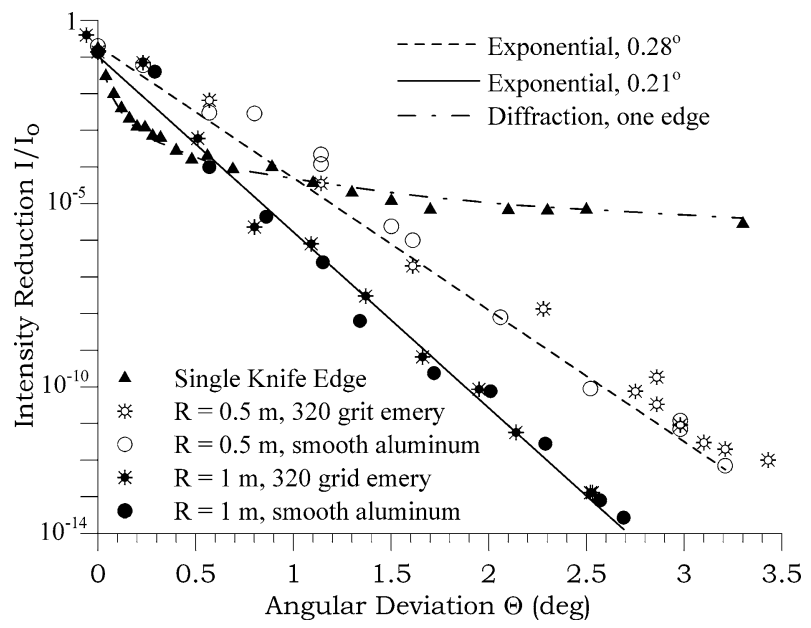

ploy a baffle system to reduce light from objects within the field of regard but beyond the field of view. The baffles of Helios, SMEI, and STEREO are multistage labyrinthine designs that employ multiple vanes to reduce light reaching the interior of the baffle aperture, and/or from diffracting over baffle edges (Buffington, Jackson, and Korendyke, 1996; Buffington, Jackson, and Hick, 2003; Eyles et al., 2009) in order to reduce stray light that 


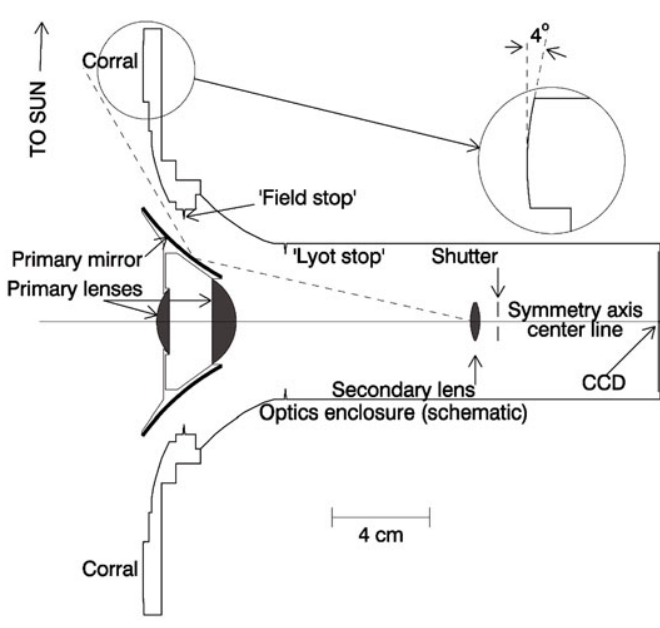

(a)

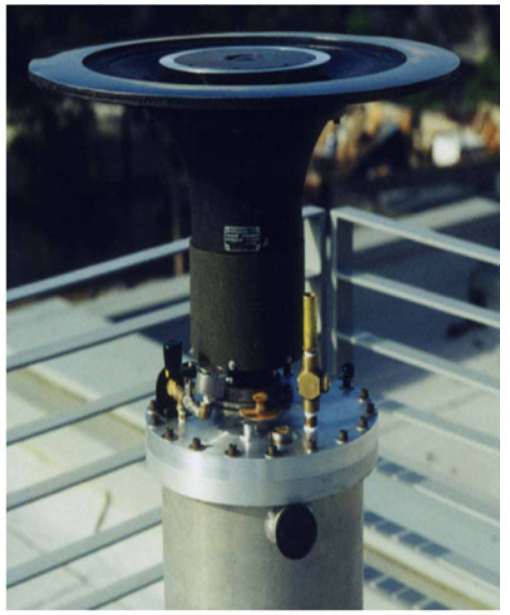

(b)

Figure 11 (a) A cross-sectional schematic diagram of a heliospheric imager (from Jackson, Buffington, and Hick, 2001). The composite optic, a combination of a mirror and lens, allows viewing over an entire $180^{\circ}$ hemisphere. The system scales; the version shown is designed to operate at $0.5 \mathrm{AU}$. The dimension becomes $8 \mathrm{~cm}$ and the angle in the upper right side of the Figure $2^{\circ}$ for an instrument at $1.0 \mathrm{AU}$. (b) A complete heliospheric imager $1 \mathrm{AU}$ model attached to the SMEI prototype CCD camera used to obtain night-sky observations in order to test instrument throughput and image quality.

could shine into the instrument aperture. It is best if an instrument defines its own field of regard, but this is only possible for a very wide-angle viewing design if an instrument location can be found on the spacecraft bus that places the instrument somewhat above a horizontal plane that extends outward from the instrument aperture, and so that the field of regard does not include the Sun or, in the case of an instrument in Earth orbit, both the Earth and the Sun.

A second restriction is placed by the brightness of the zodiacal cloud. At $1 \mathrm{AU}$, and $90^{\circ}$ elongation, its signal is from two to three orders of magnitude greater that the Thomsonscattering signal. That this signal does not vary in the same manner as the Thomsonscattering signal and can be modeled has been explored in several ways (see Buffington et al., 2009a). Measurements of the change in brightness of zodiacal-light dust bands, comet trails, and the Gegenschein (the zodiacal-dust counterglow), are studies in themselves. As distances from the Sun become less than 1 AU, the dust brightness relative to the Thomsonscattering signal decreases somewhat, and thus as shown by the Helios photometers, there is less zodiacal light to interfere with the Thomson-scattering signal. In any case, where quantitative analysis of the Thomson-scattered heliospheric light is needed, the contribution from zodiacal light must be removed. Whether this is modeled simply by determining and subtracting a minimum map of the sky brightness, or by a parametric fit to a data ensemble, or finally by employing a 3D model of the distribution and scattering of the zodiacal-dust cloud, this large zodiacal-light brightness and its removal must be accounted for in determining the upper limit of the overall detector response.

Taking all these principles into account, Buffington, Jackson, and Korendyke (1996), Buffington (1998), Buffington et al. (1998), Buffington (2000) designed a heliospheric imager that allows a view of nearly a hemisphere of sky from as close to the Sun as $2^{\circ}$ outward to as distant an elongation as $180^{\circ}$ from the solar surface (Figure 11). This instrument design employs a composite mirror-lens optical system allowing the field of view to extend over nearly an $180^{\circ}$ hemisphere (Buffington, 2000), and also employs a laboratory-tested 
diamond-turned mirror optic (Buffington et al., 2009b) that reduces residual light from bright objects present in the instrument field of view. In space free from auroral brightness, the Moon, and the Sun, and on a sunward-pointing platform, such an instrument could in theory view the whole sky photometrically with a data cadence of at least one image per hour, and weigh less than $5 \mathrm{~kg}$. Such a design requires that the spacecraft bus be tailored to meet the requirements of the instrument, in order to provide no (or at least very little) stray light from the other instruments and appendages on the spacecraft bus that must stay below the horizontal plane of the detector, and if possible outside an approximately $2^{\circ}$ field of regard greater than the $180^{\circ}$ field of view. This very small field-of-regard augmentation in angular dimension (over the field of view) is allowed because diffraction (rather than a multi-vane labyrinthine baffle system) can eliminate stray light by more than ten orders of magnitude beyond the instrument aperture (Figure 10).

\section{Summary and Conclusions}

The Helios photometers, SMEI, and the STEREO HIs all show that spaceborne imagers can operate effectively to view the Thomson-scattering brightness signal from heliospheric electrons. These instruments contribute greatly to views of the corona near the Sun, to predicting in-situ measurements from interplanetary spacecraft, and to heliospheric modeling efforts. Such data are planned using moderately-wide-angle viewing instruments on Solar Orbiter, Solar Probe, and other deep-space explorers. Here, we have presented a set of instrument specifications required for a fully-performing successful heliospheric imager, and the measurements and data-processing steps that make the best use of this remote-sensing system. When this type of instrument is properly designed and calibrated, its data are also capable of determining zodiacal-dust properties, and as a main objective it enables $3 \mathrm{D}$ reconstructions of heliospheric electron density over large volumes of the inner heliosphere.

Accurate determinations of heliospheric bulk density extend beyond just this basic heliospheric parameter. In combination with global velocity estimates (e.g. from IPS velocity remote sensing), these density measurements give an estimate of heliospheric pressure that can be used e.g. to determine the distance of the heliospheric interstellar boundary, or the dynamic solar wind pressure at Mars, and its effect on the Martian magnetosphere (Jackson et al., 2007). In combination with Faraday rotation (FR) observations, bulk densities allow the remote determination of heliospheric vector magnetic field components globally (Jensen et al., 2010). This combination of bulk density measurements combined with FR observations planned from radio arrays such as the Murchison Widefield Array (MWA) or the LOw Frequency ARray (LOFAR) now under construction may usher in a whole new remote-sensing capability.

The limits placed on heliospheric imagers from the many orders of magnitude brightness difference between the Sun and the signal to be observed requires careful design that incorporates the stray-light impacts of the instrument bus, its appendages, and other instruments on the spacecraft. Furthermore, if determining the 3D tomographic locations of heliospheric features using brightness change is considered important, the resultant need to provide photometric images that are stable over days to weeks requires an even more stringent set of specifications, as well as careful instrument calibration. The heliospheric physics community should be extremely indebted to the Helios photometer systems and to their PI Dr. Christoph Leinert for his and his colleagues' serendipitous discovery of "heliospheric plasma clouds". Without this, SMEI, and the STEREO HI instruments would have not been possible. In spite of the difficulties of such instrumentation and the requirement of much data 
analyses to make them operate effectively, heliospheric imagers thrive and find increasing uses and a place on spacecraft probes whether they are used for remote sensing only small portions of the sky as on the upcoming Solar Orbiter mission, as a proposed instrument for Solar Probe Lite (NASA AO), or for the potential measurement of rocket plumes (Dressler and Goldstein, 2010).

Acknowledgements B.V. Jackson, P.P. Hick, A. Buffington, and M.M. Bisi were supported at the University of California at San Diego by NSF grant ATM-0331513, and more recently by NSF grant ATM-0852246 and by NASA grants NAG5-134543 and NNG05GG45G. In addition, M.M. Bisi was supported by NSF grant ATM-0925023, and he and B.V. Jackson were both supported in part by AFOSR grant FA9550-06-1-0107. We acknowledge numerous useful conversations with C.J. Eyles, and N.R. Waltham. SMEI was designed and constructed by a team of scientists and engineers from the US Air Force Research Laboratory, the University of California at San Diego, Boston College, Boston University, and the University of Birmingham, UK. The STEREO/SECCHI project is an international consortium of NRL (USA), Lockheed Martin Solar and Astrophysics Lab (USA), NASA Goddard Space Flight Center (USA), RAL (UK), University of Birmingham (UK), Max-Planck-Institut für Sonnensystemforschung (Germany), Centre Spatial de Liège (CSL) (Belgium), Institut d'Optique Théorique et Appliquée (France), and the Institut d'Astrophysique Spatiale (France). The SECCHI HI instruments were developed by a collaboration that included the University of Birmingham, RAL, CSL, and NRL.

Open Access This article is distributed under the terms of the Creative Commons Attribution Noncommercial License which permits any noncommercial use, distribution, and reproduction in any medium, provided the original author(s) and source are credited.

\section{References}

Ananthakrishnan, S., Coles, W.A., Kaufman, J.J.: 1980, J. Geophys. Res. 85, 6025.

Asai, K., Kojima, M., Tokumaru, M., Yokobe, A., Jackson, B.V., Hick, P.P., Manoharan, P.K.: 1998, J. Geophys. Res. 103, 1991.

Behannon, K.W., Burlaga, L.F., Hewish, A.: 1991, J. Geophys. Res. 96, 21,213.

Billings, D.E.: 1966, A Guide to the Solar Corona, Academic Press, New York.

Bisi, M.M., Jackson, B.V., Hick, P.P., Buffington, A., Clover, J.M.: 2007, Adv. Geosci. 14, 161.

Bisi, M.M., Jackson, B.V., Hick, P.P., Buffington, A., Odstrcil, D., Clover, J.M.: 2008a, J. Geophys. Res. 113, A00A11.

Bisi, M.M., Jackson, B.V., Buffington, A., Hick, P.P., Manoharan, P.K., Clover, J.M.: 2008b, Adv. Geosci. 21, 33.

Bisi, M.M., Jackson, B.V., Buffington, A., Clover, J.M., Hick, P.P., Tokumaru, M.: 2009a, Solar Phys. 256, 201.

Bisi, M.M., Jackson, B.V., Clover, J.M., Manoharan, P.K., Tokumaru, M., Hick, P.P., Buffington, A.: 2009b, Ann. Geophys. 27, 4479.

Brueckner, G.E., Howard, R.A., Koomen, M.J., Korendyke, C.M., Michels, D.J., Moses, J.D., Socker, D.G., Dere, K.P., Lamy, P.L., Llebaria, A., Bout, M.V., Schwenn, R., Simnett, G.M., Bedford, D.K., Eyles, C.J.: 1995, Solar Phys. 162, 357.

Buffington, A.: 1998, Appl. Opt. 37, 4284.

Buffington, A.: 2000, Appl. Opt. 39, 2683.

Buffington, A., Jackson, B.V., Korendyke, C.M.: 1996, Appl. Opt. 35, 6669.

Buffington, A., Jackson, B.V., Hick, P.P.: 2003, Proc. SPIE 4853, 490.

Buffington, A., Jackson, B.V., Hick, P.P.: 2005, Proc. SPIE 5901, 590118,1.

Buffington, A., Hick, P., Jackson, B.V., Korendyke, C.M.: 1998, Proc. SPIE 3442, 77.

Buffington, A., Band, D.L., Jackson, B.V., Hick, P.P., Smith, A.C.: 2006, Astrophys. J. 637, 880.

Buffington, A., Morrill, J.S., Hick, P.P., Howard, R.A., Jackson, B.V., Webb, D.F.: 2007, Proc. SPIE 6689, 66890B, 1 .

Buffington, A., Bisi, M.M., Clover, J.M., Hick, P.P., Jackson, B.V., Kuchar, T.A.: 2008, Astrophys. J. 677, 798.

Buffington, A., Bisi, M.M., Clover, J.M., Hick, P.P., Jackson, B.V., Kuchar, T.A., Price, S.D.: 2009a, Icarus 203, 124.

Buffington, A., Bach, K.G., Bach, B.W., Bach, E.K., Bisi, M.M., Hick, P.P., Jackson, B.V., Klupar, P.D.: 2009b, Proc. SPIE 7438, 743800. 
Cox, A.N.: 2000, Allen's Astrophysical Quantities, 4th edn. AIP Press, New York.

Davis, C.J., Davies, J.A., Lockwood, M., Rouillard, A.P., Eyles, C.J., Harrison, R.A.: 2009, Geophys. Res. Lett. 36, 08102.

Dressler, R., Goldstein, N.: 2010, AFRL Technical Report AFRL-RV-HA-TR-2010, in press.

Eyles, C.J., Simnett, G.M., Cooke, M.P., Jackson, B.V., Buffington, A., Hick, P.P., Waltham, N.R., King, J.M., Anderson, P.A., Holladay, P.E.: 2003, Solar Phys. 217, 319.

Eyles, C.J., Harrison, R.A., Davis, C.J., Waltham, N.R., Shaughnessy, B.M., Mapson-Menard, H.C.A., Bewsher, D., Crothers, S.R., Davies, J.A., Simnett, G.M., Howard, R.A., Moses, J.D., Newmark, J.S., Socker, D.G., Halain, J.P., Defise, J.M., Mazy, E., Rochus, P.: 2009, Solar Phys. 254, 387.

Fry, C.D., Dryer, M., Smith, Z., Sun, W., Deehr, C.S., Akasofu, S.-I.: 2003, J. Geophys. Res. 108(A2), 1070. Gapper, G.R., Hewish, A., Purvis, A., Duffett-Smith, P.J.: 1982, Nature 296, 633.

Hansen, R.T., Garcia, C.J., Grognard, R.R.-M., Sheridan, K.V.: 1971, Proc. Astron. Soc. Austr. $2(1), 57$.

Hansen, R., Garcia, C.J., Hansen, S.F., Yasukawa, E.: 1974, Publ. Astron. Soc. Pac. 86, 500.

Harrison, R.A., Davis, C.J., Eyles, C.J., Bewsher, D., Crothers, S., Davies, J.A., Howard, R.A., Moses, D.J., Socker, D.G., Halain, J.-P., Defise, J.-M., Mazy, E., Rochus, P., Webb, D.F., Simnett, G.M.: 2008, Solar Phys. 247, 171.

Harrison, R.A., Davies, J.A., Roulliard, A.P., Davis, C.J., Eyles, C.J., Bewsher, D., Crothers, S.R., Howard, R.A., Sheeley, N.R., Vourlidas, A., Webb, D., Brown, D.S., Dorrian, G.: 2009, Solar Phys. 256, 219.

Hewish, A., Bravo, S.: 1986, Solar Phys. 106, 185.

Hewish, A., Scott, P.F., Wills, D.: 1964, Nature 203, 1214.

Houminer, Z.: 1971, Nature Phys. Sci. 231, 165.

Howard, T.A., Tappin, S.J.: 2009, Space Sci. Rev. 147, 31.

Howard, R.A., Moses, J.D., Vourlidas, A., Newmark, J.S., Socker, D.G., Plunkett, S.P., Korendyke, C.M., Cook, J.W., Hurley, A., Davila, J.M., Thompson, W.T., St. Cyr, O.C., Mentzell, E., Mehalick, K., Lemen, J.R., Wuelser, J.P., Duncan, D.W., Tarbell, T.D., Wolfson, C.J., Moore, A., Harrison, R.A., Waltham, N.R., Lang, J., Davister, C., Eyles, C.J., Mapson-Menard, H., Simnett, G.M., Halain, J.P., Defise, J.M., Mazy, E., Rochus, P., Mercier, R., Ravet, M.F., Delmotte, F., Auchere, F., Delaboudinière, J.-P., Bothmer, V., Deutsch, W., Wang, D., Richel, N., Cooper, S., Stephens, V., Maahs, G., Baugh, R., Mcmullin, D., Carter, T.: 2008, Space Sci. Rev. 136, 67.

Jackson, B.V.: 1985a, Solar Phys. 95, 363.

Jackson, B.V.: 1985b, Solar Phys. 100, 563.

Jackson, B.V.: 1991, J. Geophys. Res. 96, 11,307.

Jackson, B.V., Leinert, C.: 1985, J. Geophys. Res. 90, $10,759$.

Jackson, B.V., Froehling, H.R.: 1995, Astron. Astrophys. 299, 885.

Jackson, B.V., Hick, P.P.: 2002, Solar Phys. 211, 344.

Jackson, B.V., Hick, P.P.: 2005, In: Gary, D.G., Keller, C.U. (eds.) Solar and Space Weather Radiophysics: Current Status and Future Developments, Astrophys. Space Scien. Lib. 314, Kluwer Academic, Dordrecht, 355.

Jackson, B.V., Rompolt, B., Švestka, Z.: 1988, Solar Phys. 115, 327.

Jackson, B.V., Buffington, A., Hick, P.P.: 2001, In: Marsch, E., Marinez Pillet, V., Fleck, B., Marsden, R. (eds.) Solar Encounter: the First Solar Orbiter Workshop SP-493, ESA, Noordwijk, 251.

Jackson, B.V., Hick, P.P., Buffington, A.: 2002, Proc. SPIE, Waikoloa 4853, 23.

Jackson, B.V., Howard, R.A., Sheeley, N.R., Jr., Michels, D.J., Koomen, M.J., Illing, R.M.E.: 1985, J. Geophys. Res. 90, 5075.

Jackson, B.V., Hudson, H.S., Nichols, J.D., Gold, R.E.: 1989, In: Waite, J.H., Jr., Burch, J.L., Moore, R.L. (eds.) Solar System Plasma Physics, Geophysical Monograph 54, 291.

Jackson, B.V., Hick, P.L., Kojima, M., Yokobe, A.: 1998, J. Geophys. Res. 103, 12,049.

Jackson, B.V., Hick, P.P., Buffington, A., Kojima, M., Tokumaru, M., Fujiki, K., Ohmi, T., Yamashita, M.: 2003, In: Velli, M., Bruno, R., Marara, F. (eds.) Proc. Solar Wind X, AIP Conference Proc. 679, 75.

Jackson, B.V., Buffington, A., Hick, P.P., Altrock, R.C., Figueroa, S., Holladay, P.E., Johnston, J.C., Kahler, S.W., Mozer, J.B., Price, S., Radick, R.R., Sagalyn, R., Sinclair, D., Simnett, G.M., Eyles, C.J., Cooke, M.P., Tappin, S.J., Kuchar, T., Mizuno, D., Webb, D.F., Anderson, P.A., Keil, S.L., Gold, R.E., Waltham, N.R.: 2004, Solar Phys. 225, 177.

Jackson, B.V., Buffington, A., Hick, P.P., Wang, X., Webb, D.: 2006, J. Geophys. Res. 111, A04S91.

Jackson, B.V., Boyer, J.A., Hick, P.P., Buffington, A., Bisi, M.M., Crider, D.H.: 2007, Solar Phys. 241(2), 385.

Jackson, B.V., Bisi, M.M., Hick, P.P., Buffington, A., Clover, J.M., Sun, W.: 2008a, J. Geophys. Res. 113, A00A15.

Jackson, B.V., Hick, P.P., Buffington, A., Bisi, M.M., Clover, J.M., Tokumaru, M.: 2008b, Adv. Geosci. 21, 339.

Jackson, B.V., Hick, P.P., Buffington, A., Bisi, M.M., Clover, J.M.: 2009, Ann. Geophys. 27, 4097. 
Jackson, B.V., Hick, P.P., Buffington, A., Bisi, M.M., Clover, J.M., Tokumaru, M., Fujiki, K.: 2010, In: Maksimovic, M., Issautier, K., Meyer-Vernet, N., Moncuquet, M., Pantellini, F. (eds.) Proc. Solar Wind 12, AIP. Conf. Proc. 1216, 659.

Jensen, E.A., Bisi, M.M., Mulligan, T.L., Clover, J.M., Hick, P.P., Jackson, B.V.: 2010, Solar Phys., this issue. doi:10.1007/s11207-010-9543-2.

Kaiser, M.L., Kucera, T.A., Davila, J.M., St. Cyr, O.C., Guhathakurta, M., Christian, E.: 2008, Space Sci. Rev. 136, 5.

Kojima, M., Tokumaru, M., Watanabe, H., Yokobe, A., Asai, K., Jackson, B.V., Hick, P.L.: 1998, J. Geophys. Res. 103, 1981.

Koomen, M.J., Detwiler, C.R., Brueckner, G.E., Cooper, H.W., Tousey, R.: 1975, Appl. Opt. 14, 743.

Leinert, C., Klüppelberg, D.: 1974, Appl. Opt. 13, 556.

Leinert, C., Pitz, E.: 1989, Astron. Astrophys. 210, 399.

Leinert, C., Richter, I., Planck, B.: 1982, Astron. Astrophys. 110, 111.

Leinert, C., Link, H., Pitz, E., Salm, N., Klüppelberg, D.: 1975, Raumfahartforschung 19, 264.

Leinert, C., Pitz, E., Link, H., Salm, N.: 1981, J. Space Sci. Instrum. 5, 257.

Lugaz, N., Vourlidas, A., Roussev, I.I., Jacobs, C., Manchester IV, W.B., Cohen, O.: 2008, Astrophys. J. Lett. 684, 111.

Liu, Y., Davies, J.A., Luhmann, J.G., Vourlidas, A., Bale, S.D., Lin, R.P.: 2010, Solar Phys., submitted.

MacQueen, R.M., Eddy, J.A., Gosling, J.T., Hildner, E., Munro, R.H., Newkirk, G.A., Jr., Poland, A.I., Ross, C.L.: 1974, Astrophys. J. Lett. 187, 85.

Mizuno, D.R., Buffington, A., Cooke, M.P., Eyles, C.J., Hick, P.P., Holladay, P.E., Jackson, B.V., Johnston, J.C., Kuchar, T.A., Mozer, J.M., Price, S.D., Radick, R.R., Simnett, G.M., Sinclair, D., Webb, D.F.: 2005, J. Geophys. Res. 110, A07230.

Moran, T.G., Davila, J.M.: 2004, Science 305(5680), 66.

Morrill, J.S., Korendyke, C.M., Andrews, M., Biesecker, D., Brueckner, G.E., Esfandiari, E., Giovane, F., Howard, R.A., Koomen, M., Lamy, P., Llebaria, A., Michels, D., Moses, D., Plunkett, S.P., Rich, N., Thernisien, A.F., Vourlidas, A., Wang, D.: 2006, Solar Phys. 233, 331.

Munro, R.H.: 1977. In: Topical Conference on Solar and Interplanetary Physics, Tucson, Arizona, January 12-15, 10 .

Odstrcil, D., Pizzo, V.J.: 1999, J. Geophys. Res. 104, 483.

Odstrcil, D., Pizzo, V.J., Linker, J.A., Riley, P., Lionello, R., Mikic, Z.: 2004, J. Atmos. Solar-Terr. Phys. 66, 1311.

Ogilvie, K.W., Desch, M.D.: 1997, Adv. Space Res. 20, 559.

Porsche, H. (ed.): 1984, 10 Jahre HELIOS, Festschrift aus Anlaß des 10. Jahrestages des Starts der Sonnensonde Helios am 10. Dezember 1974, DFVLR Oberpfaffenhofen.

Richter, I., Leinert, C., Planck, B.: 1982, Astron. Astrophys. 110, 115.

Reiner, M.J., Jackson, B.V., Webb, D.F., Mizuno, D.R., Kaiser, M.L., Bougeret, J.L.: 2005, J. Geophys. Res. 110(A9), A09S14.

Rouillard, A.P., Davies, J.A., Forsyth, R.J., Davis, C.J., Harrison, R.A., Lockwood, M., Bewsher, D., Crothers, S., Eyles, C.J., Hapgood, M.A., Perry, C.H.: 2008, Geophys. Res. Lett. 35, 10110.

Sheeley, N.R., Jr., Michels, D.J., Howard, R.A., Koomen, M.J.: 1980, Astrophys. J. Lett. 237, 99.

Sheeley, N.R., Herbst, A.D., Palatchi, C.A., Wang, Y.-M., Howard, R.A., Moses, J.D., Vourlidas, A., Newmark, J.S., Socker, D.G., Plunkett, S.P., Korendyke, C.M., Burlaga, L.F., Davila, J.M., Thompson, W.T., St. Cyr, O.C., Harrison, R.A., Davis, C.J., Eyles, C.J., Halain, J.P., Wang, D., Rich, N.B., Battams, K., Esfandiari, E., Stenborg, G.: 2008, Astrophys. J. 675, 853.

Socker, D.G., Howard, R.A., Korendyke, C.M., Simnett, G.M., Webb, D.F.: 2000, Proc. SPIE 4139, 284.

Tappin, S.J.: 2006, Solar Phys. 233, 233.

Tappin, S.J., Howard, T.A.: 2009, Space Sci. Rev. 147, 55.

Tappin, S.J., Buffington, A., Cooke, M.P., Eyles, C.J., Hick, P.P., Holladay, P.E., Jackson, B.V., Johnston, J.C., Kuchar, T., Mizuno, D., Mozer, J.B., Price, S., Radick, R.R., Simnett, G.M., Sinclair, D., Waltham, N.R., Webb, D.F.: 2004, Geophys. Res. Lett. 31, 2802.

Tokumaru, M., Kojima, M., Fujiki, K., Yamashita, M., Jackson, B.V.: 2007, J. Geophys. Res. 112, A05106.

Tokumaru, M., Kojima, M., Fujiki, K.: 2010, J. Geophys. Res 115, A04102.

Tousey, R.: 1973, In: Rycroft, M.J., Runcorn, S.K. (eds.) Space Res. 8, Akademie-Verlag, Berlin, 713.

Vourlidas, A., Howard, R.A.: 2006, Astrophys. J. 642, 1216.

Webb, D.F., Mizuno, D.R., Buffington, A., Cooke, M.P., Eyles, C.J., Fry, C.D., Gentile, L.C., Hick, P.P., Holladay, P.E., Howard, T.A., Hewitt, J.G., Jackson, B.V., Johnston, J.C., Kuchar, T.A., Mozer, J.B., Price, S., Radick, R.R., Simnett, G.M., Tappin, S.J.: 2006, J. Geophys. Res. 111, A12101.

Webb, D.F., Howard, T.A., Fry, C.D., Kuchar, T.A., Odstrcil, D., Jackson, B.V., Bisi, M.M., Harrison, R.A., Morrill, J.S., Howard, R.A., Johnston, J.C.: 2009a, Solar Phys. 256, 239.

Webb, D.F., Howard, T.A., Fry, C.D., Kuchar, T.A., Mizuno, D.R., Johnston, J.C., Jackson, B.V.: 2009b, Space Weather 7, S05002. 